\title{
CD22 Positive
}

National Cancer Institute

\section{Source}

National Cancer Institute. CD22 Positive. NCI Thesaurus. Code C131066.

Indicates that CD22 expression has been detected in a sample. 